\title{
Horizon Picking in 3D Seismic Images
}

\author{
Maria Faraklioti ${ }^{1}$ and Maria Petrou $^{1,2}$ \\ 1 University of Surrey, Guildford, GU2 7XH, UK \\ 2 The Institute of Telematics and Informatics, CERTH, \\ PO Box 361, Thermi, Thessaloniki, Greece \\ M.Petrou@surrey.ac.uk
}

\begin{abstract}
In this paper, we present an automatic horizon picking algorithm, based on a surface detection technique, to detect horizons in $3 \mathrm{D}$ seismic data. The surface detection technique, and the use of 6 connectivity, allows us to detect fragments of horizons which are afterwards combined to form full horizons. The criteria of combining the fragments are similarity of orientation of the fragments, as expressed by their normal vectors, and proximity using 18-connectivity. The identified horizons are interrupted at faults, as required by the experts.
\end{abstract}

\section{Introduction}

Volumetric seismic image analysis provides more complete information about features such as faults, horizons and mounds in the Earth's subsurface, than slice by slice $2 \mathrm{D}$ analysis.

Horizons are surfaces which separate different rock layers in depositional environments characterised by different reflection properties. Horizons are often interrupted by faults which are usually vertical fractures that cause displacement of the horizons. Generally, horizons are represented by curves in 2D seismic images, and by surfaces in 3D data. So, we consider it inappropriate to treat horizons as edges for their detection [1], [2]. Several researchers propose line detection algorithms to detect and label horizons in 2D seismic images [8],[9],[10], [5]. 3D horizon picking is a difficult, more complex and more computationally demanding task. Some algorithms have recently been developed for 3D horizon picking that either detect horizons as the union of small 3D seismic surfaces [3] or model them as triangulated surfaces [4]. In this paper, we present an automatic algorithm for identifying the seismic horizons as the mid surfaces of the bright (or dark) layers in the seismic volume data by using an image analysis technique. Connected component analysis is applied and small horizon fragments are identified this way. The formation of large horizons is achieved by joining fragments which have consistent orientation and proximity. In this way, large horizon fragments which stop at discontinuities are constructed.

\section{Methodology}

The original candidate horizon voxels are picked up as the extrema of the second derivative of a Gaussian filter which are surrounded by two extrema of the 
opposite sign, along the vertical direction [6]. The size of the filter used for this job is most crucial. The filter should be tuned to the width of the horizons [6], but at the same time it should not be too wide in order to avoid the interference from nearby horizons. We modelled the succession of horizons as a series of square waves, and computed the optimal relationship between the standard deviation $\sigma$ of the Gaussian filter used and the width of each pulse. This stylised approximation yielded that in order to maximise the response of the filter to such a pulse while at the same time we minimise the response of the filter to neighbouring pulses, we must choose $\sigma$ so that $\sigma \simeq 1.12 d$. The average value of $d$ was computed as the average distance between successive zero crossings along various columns of the seismic data at our disposal and it was found to be roughly 3 . The mode of the distribution of $d$ was 3 . This pointed at a filter with $\sigma \simeq 3$. In practice narrower filters appeared to work better and in particular filters with $\sigma$ in the range [1.5,2.8]. So, we chose a filter with $\sigma=2$, of size 13 taps.

An example volume of seismic data is shown in figure 1 where for displaying volumes the TriTex visualisation package is used [7].

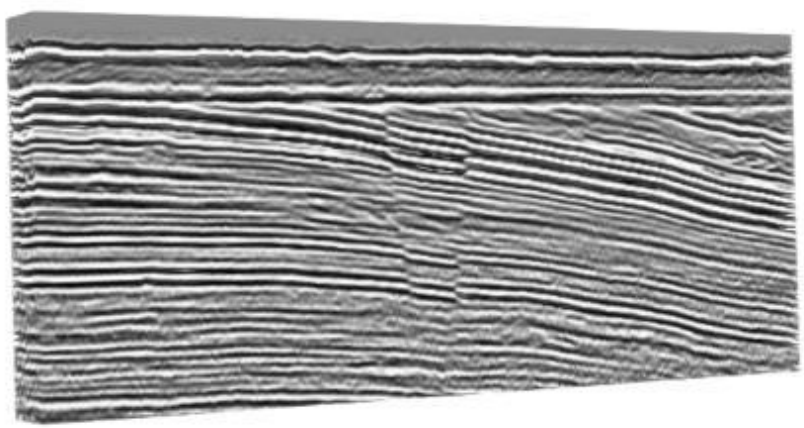

Fig. 1. 3D seismic data

The identified candidate horizon voxels were labelled using connected component analysis. In 3D data one may choose between 6-, 18- or 26-connectivity. The horizons produced by 6 connectivity were too fragmented while 18 and 26 connectivity produced composite "horizons". We used 6 connectivity to produce fragments of horizons which will be subsequently combined to form full horizons.

As the horizons are essentially flat surfaces, fragments of the same horizon must be roughly at the same distance from the origin and have similar orientation, i.e. their normals must be parallel. Figure 2 shows an example of a small fragment.

Let $C$ be the centre of gravity of the fragment, computed by taking the average position of all voxels that make up the fragment $(\bar{x}, \bar{y}, \bar{z})$. We can compute 


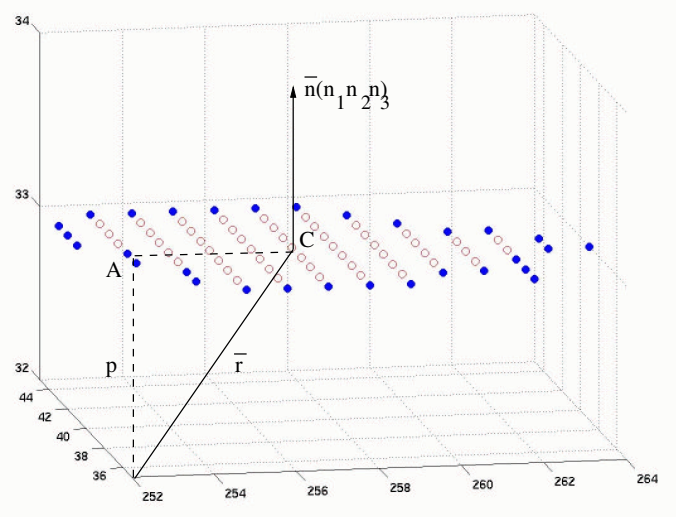

Fig. 2. A horizon fragment. Filled points are the border points

the least square error fitting plane through the fragment voxels by finding the eigenvectors of the covariance matrix of the voxels that make up the fragment. The least square error plane is defined by the eigenvectors that correspond to the two largest eigenvalues and the normal to the plane coincides with the eigenvector with the smallest eigenvalue. Let $\bar{r}$ be the position vector of $C$ and $\bar{n}$ the normal vector to the fragment (see figure 2). The distance $p$ of the origin of the axes from the fragment is equal to the projection of vector $\bar{r}$ on the normal vector $\bar{n}$ :

$$
p=\bar{r} \cdot \bar{n}
$$

Fragments that have equal distance $p$ and equal normal vectors (within a certain tolerance), probably belong to the same horizon so, they may be considered for merging. However, they should not be merged unless they are next to each other. To check for proximity we identify first the border voxels of each fragment. These are the voxels of the fragment which within the $2 D$ horizon plane have neighbouring voxels with different labels. These voxels are highlighted as filled circles in the example fragment of figure 2. Two fragments may be merged only if some border voxels of one are neighbours of some border voxels of the other. As the fragments were created using 6-connectivity, it is obvious that the relationship "is neighbour of" will be void unless we increase the connectivity. Thus, to identify fragments with neighbouring boundary voxels, 18-connectivity is used.

To decide upon the similarity of the $\bar{n}$ values between fragments, we put all fragments into bins of size $\Delta n_{x} \times \Delta n_{y} \times \Delta n_{z}$ where $\Delta n_{x}, \Delta n_{y}$ and $\Delta n_{z}$ is the tolerance with which the values of the components of vectors $\bar{n}$ will be judged to be equal. Fragments that fall in the same bin will be considered for merging. To avoid bin border effects, as for example two fragments with very similar values 
of $\bar{n}$ may fall in neighbouring bins, candidate fragments for merging are not only fragments that belong to the same bin but also fragments that belong to neighbouring bins, in the 3 dimensional histogram of normal vectors.

Since $p$ depends on the value of $\bar{n}$ and the position of the gravity centre $C$, the tolerance in $p$ depends on the tolerance of $\bar{n}$. More specifically, from equation 1 it follows that:

$$
p=\bar{x} n_{x}+\bar{y} n_{y}+\bar{z} n_{z}
$$

Then, to the first approximation, the errors in the value of $p$ due to errors in the individual components of the gradient vector (assuming that the centre of gravity of the fragment is estimated with no error), are

$$
\Delta p_{x}=\bar{x} \Delta n_{x}, \quad \Delta p_{y}=\bar{y} \Delta n_{y}, \quad \Delta p_{z}=\bar{z} \Delta n_{z}
$$

In the worse case these errors may all add up to the same direction, but this is very unlikely. As an estimate in the error in the value of $p$ (and therefore an estimate of the tolerance with which values of $p$ should be matched), we use the average of the above three errors:

$$
\Delta p=\frac{\bar{x} \Delta n_{x}+\bar{y} \Delta n_{y}+\bar{z} \Delta n_{z}}{3}
$$

Two fragments may be considered for merging if their absolute distance difference is less than the average of their tolerance values, that is:

$$
\left|p_{1}-p_{2}\right|<\frac{\Delta p_{1}+\Delta p_{2}}{2}
$$

So, the criteria for merging two fragments are the following:

- the fragments belong to the same or neighbouring orientation bins;

- they have similar distance from the origin within a tolerance $\Delta p$ computed as in (5);

- at least one border voxel of one fragment is in the 18-neighbourhood of the border voxels of the other fragment.

If these criteria are met then the fragments are merged and labelled with the same label. After each merge, the gravity centre, the distance $p$, the normal vector $\bar{n}$ and the border voxels of the combined fragment are computed. The merging process continues until no more merges can take place.

Clearly, the choice of bin size for the three parameters, $n_{x}, n_{y}$ and $n_{z}$, affects the final number of merges. The components of the normal vector $n_{x}, n_{y}$ and $n_{z}$ are the cosines of the angle the normal vector forms with each of the axes. So, a tolerance in $\bar{n}$ corresponds to a tolerance in the corresponding relative orientation between the two planes.

For example, if fragments that differ in slope by about $1^{\circ}$ are to be considered for merging, then the bin size for the components of $\bar{n}$ should be 0.02 . As the angle bin increases, more fragments are merged as expected although the bin size range is restricted by the separability of the formed horizons. Several experiments were conducted by varying the angle bin sizes. 


\section{Experimental results}

Horizon picking on the cube shown in figure 1 produced the horizons shown in figure 3. The connected component analysis on the detected horizons resulted

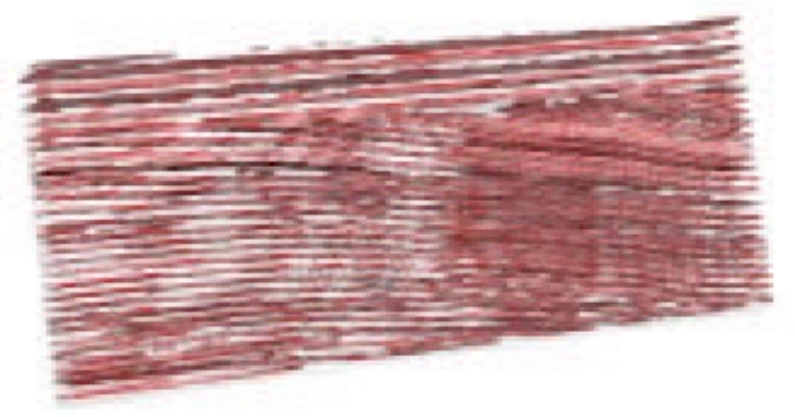

Fig. 3. Output of the filtering process

in 3037 different labelled fragments. These were reduced to 1468 by removing fragments of 50 or fewer voxels. Table 1 shows the number of final horizons produced after the merging process for different tolerance in the values of the gradient vector components. The corresponding tolerance $\Delta \theta$ in gradient vector orientation is also shown. When this tolerance becomes more than $8^{\circ}$, the merging procedure produces composite horizons. The 40 largest merged horizon

Table 1. Bin size for each parameter and remaining labels out of 1468

\begin{tabular}{cccccc}
\hline no & $\Delta n_{x}$ & $\Delta n_{y}$ & $\Delta n_{z}$ & $\Delta \theta$ & remaining labels \\
\hline 1 & 0.02 & 0.02 & 0.02 & $1^{o}$ & 670 \\
2 & 0.04 & 0.04 & 0.04 & $3^{o}$ & 485 \\
3 & 0.08 & 0.08 & 0.08 & $5^{o}$ & 298 \\
4 & 0.1 & 0.1 & 0.1 & $7^{\circ}$ & 264 \\
5 & 0.13 & 0.13 & 0.13 & $8^{o}$ & 217 \\
\hline
\end{tabular}

fragments from case 5 are also shown in 3D in figure 4 . As it can be observed, most of the labelled horizons stop at the position of the fault. 


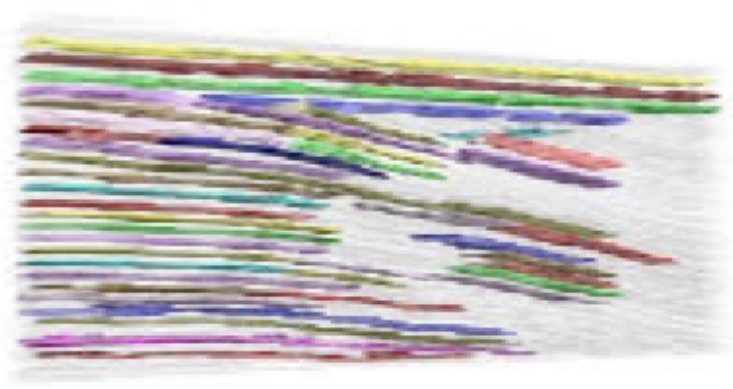

Fig. 4. 40 largest horizons which resulted from the merging process obtained with the parameters shown in the last row of table 1.

\section{Conclusions}

Our task was to identify layers created from deposition of various sediments over time called horizons, by using image analysis techniques on 3D data. We used the second derivative of a Gaussian filter to extract fragments of horizons which were subsequently merged using some criteria of coplanarity within certain tolerance. The algorithm outputs larger horizon fragments differently labelled, which stop at faults.

The implementation of the algorithm is easy since it only involved convolution of the image in three directions with the Gaussian filter. The algorithm needs no preprocessing or training. The only input is the filter size and the tolerance in normal vector orientation for horizon fragments that are to be merged.

Acknowledgements: This work was supported by the TriTex project funded by the European Commission, (Project IST-1999-20500). Many thanks to Schlumberger Stavanger Research and Seismic Area C (Norsk Hydro, Statoil, Norsk Agip and Fortum Petroleum) for the provision of data and to Fraunhofer Institute for Applied Information Technology FIT for the provision of the visualisation package.

\section{References}

1. I. Bondar. Seismic horizon detection using image processing algorithms. Geophysical Prospecting, 40:785-800, 1992.

2. N. Keskes, A. Boulanouar, and O. Faugeras. Application of image analysis techniques to seismic data. Proceedings of the IEEE International Conference on Acoustics, Speech and Signal Processing, pages 855-858, 1982.

3. N. Keskes, P. Zaccagnino, D. Rether, and P. Mermey. Automatic extraction of 3-D seismic horizons. In Society of Exploration Geophysicists(SEG), Annual Meeting Expanded Abstracts, pages 557-559, 1983. 
4. P. Lavest and Y. Chipot. Building complex horizons for 3-D seismic. In Society of Exploration Geophysicists(SEG), Annual Meeting Expanded Abstracts, pages 159$161,1993$.

5. C. S. Maroni, A. Quinquis, and S. Vinson. Horizon picking on subbottom profiles using multiresolution analysis. Digital Signal Processing, 11:269-287, 2001.

6. M. Petrou. Optimal convolution filters and an algorithm for the detection of wide linear features. IEE Proceedings, 140:331-339, 1993.

7. S. Pieper and T. Berlage. Enhancing volume visualization of $3-\mathrm{D}$ seismic data by exploitation of 3-D texture features. In 64th European Association of Geoscientists and Engineers(EAGE) Annual Conference and Exhibition, 2002.

8. I. Pitas and C. Kotropoulos. A texture-based approach to the segmentation of seismic images. Pattern Recognition, 25:929-945, 1992.

9. I. Pitas and A.N. Venetsanopoulos. AGIS: An expert system for automated geophysical interpretation of seismic images. Proceedings of the IEEE International Conference on Acoustics, Speech and Signal Processing, pages 2256-2259, 1987.

10. V. Roberto, A. Peron, and P.L. Fumis. Low-level processing techniques in geophysical image interpretation. Pattern Recognition Letters, 10:111-122, 1989. 